\section{Dermatofibrosarcoma protuberans diagnosed by a single biopsy}

\author{
Kristian Bakke Arvesen, ${ }^{1}$ \\ Carsten Sauer Mikkelsen, ${ }^{1}$ \\ Torben Steiniche, ${ }^{2}$ \\ Birgitte Stausbol-Gron ${ }^{3}$ \\ 'Dermatology Clinic, Bronderslev; \\ 2Department of Pathology and \\ ${ }^{3}$ Dermatology, Aarhus University \\ Hospital, Denmark
}

\section{Abstract}

This brief report is about a 9 year old girl presenting with a $2.5 \mathrm{~cm}$ circular blue to violet discoloration on the anterolateral upper left thigh. The first biopsy taken revealed the diagnosis dermatofibrosarcoma protuberans. The patient underwent surgery at a specialized sarcoma center with post operative histology showing free wide resection margin. Preoperative chest $\mathrm{x}$-ray showed no sign of metastasis. This brief report emphasizes the significance of the use of biopsy when cutaneous elements look suspicious and diagnosis is unclear.

\section{Case Report}

A 9 year old, previously healthy girl, presented with a $2.5 \mathrm{~cm}$ circular, blue to violet, slightly elevated discoloration on the upper anterolateral left thigh (Figure 1). Two years prior to the

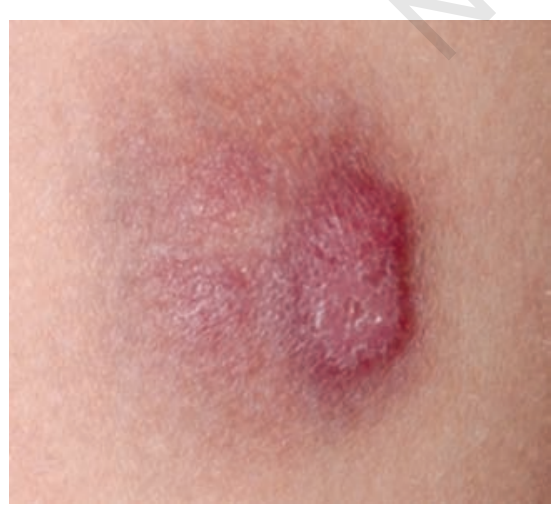

Figure 1. The clinical presentation of the cutaneous thickening with violet discoloration on the anterolateral upper left thigh, just before the biopsy was taken. consultation she had a minor trauma against the area and the described cutaneous changes developed shortly after. Her general physician suspected a foreign body and conducted an ultrasound of the thigh. All findings were normal except for a $6 \mathrm{~mm}$ fluid accumulation. In addition, the patient's own GP tried treatment with topical steroid without success. The patient was then advised to seek further dermatological investigation.

Dermatological examination revealed the earlier described intumescens at the thigh. Except for minor tenderness when palpating the discoloration, the examination was normal. A skin biopsy was taken and histologically, the dermis was replaced by a tumor composed of interwoven bundles of rather uniform, small spindle-cells (Figure 2). Staining with CD34 showed that the tumor cells characteristically expressed CD34 (Figure 3). Thus, histology revealed the diagnosis dermatofibrosarcoma protuberans grade I.

The patient was referred to the specialized sarcoma center to the further treatment starting with a preoperative chest $\mathrm{x}$-ray. The $\mathrm{x}$-ray showed no signs of metastasis. The tumor was removed using wide local excision, with postoperative histology showing wide free resection margin. The patient is now set up to annual clinical controls during the next 5 years to detect any recurrence.

\section{Discussion}

Dermatofibrosarcoma protuberans (DFSP) is a relatively rare tumor with only $0.8-5$ cases per million/year. ${ }^{1}$ The localization of this type of cancer is $50 \%$ on the thorax, $40 \%$ on the limbs, $10 \%$ head/neck area. ${ }^{1}$ The clinical pres-

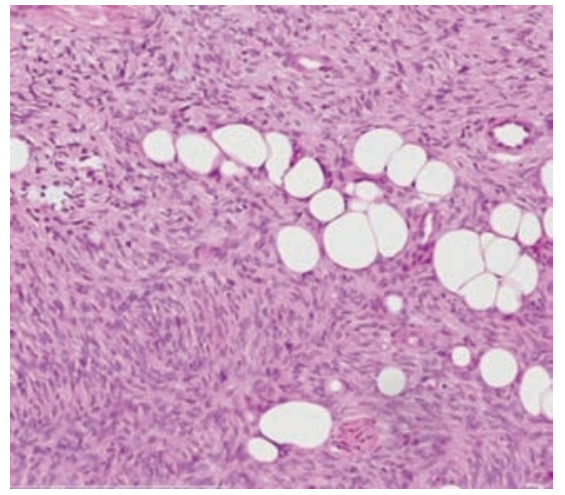

Figure 2. The dermis is replaced by a tumor composed of interwoven bundles of rather uniform, small spindle-cells. The tumor cells insinuate between fat cells in the subcutis (Hematoxylin and Eosin).
Correspondence: Kristian Bakke Arvesen, Skt.joergensgade 11, 7-5, 9000 Aalborg, Denmark. Tel. +47.9263.7999.

E-mail: kristian.bakke.arvesen@gmail.com

Key words: dermatofibrosarcoma protuberans, thigh, biopsy.

Contributions: KBA, CSM are the main authors; BSG, author, clinical photo, connecting work between departments; TS, histology.

Conflict of interests: the authors report no potential conflict of interests.

Received for publication: 14 may 2012.

Revision received: 7 June 2012.

Accepted for publication: 15 June 2012.

This work is licensed under a Creative Commons Attribution NonCommercial 3.0 License (CC BYNC 3.0).

CC Copyright K.B. Arvesen et al., 2012

Licensee PAGEPress, Italy

Dermatology Reports 2012; 4:e10

doi:10.4081/dr.2012.e10

entation of DFSPs are in the early stages plaque like areas of cutaneous thickening with violet/blue or red discoloration. When the tumor slowly enlarges it becomes raised, firm and nodular and the surrounding skin may become teleangieactatic. DFSP is low malignant and a locally aggressive growing cancer, only $1-4 \%$ disseminates. ${ }^{1}$ The dissemination is hematogenic to lungs and bones. ${ }^{2}$ Sentinel node biopsy is not recommended because of

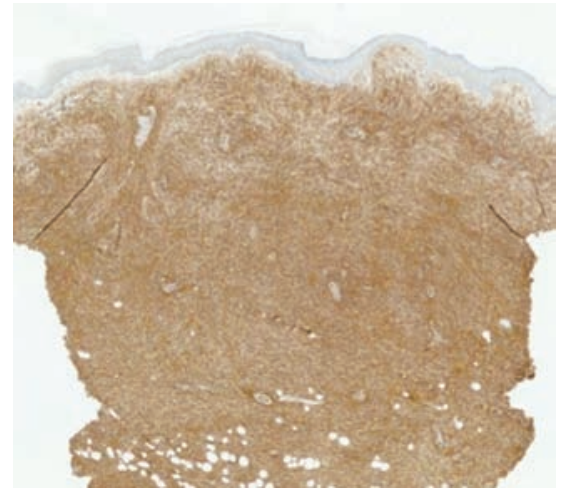

Figure 3. The tumor cells characteristically express CD34. 
the seldom lymph node involvement $(<1 \%)$. The mortality is high with metastasis, the majority of patients die within the 2 first years. ${ }^{1}$ Without metastasis and with total surgical tumor resection, the prognosis is good with 5 year survival rate of $93-100 \% .^{1-4}$ The recurrence rate is up to $50-75 \%$, most of them within 3 years..$^{1-3}$

The treatment for DFSP is tumor resection with pathologically negative margins. The preferred margin is $3-4 \mathrm{~cm}^{1-4}$ This is called wide resection. For the surgeon, Mohs surgery was not in consideration, since en bloc resection is preferred in sarcomas. Furthermore, the DFSP has infiltrating elements that are hard to detect during histology. So margins have to be large, at least some centimetres.

\section{Conclusions}

A new kind of treatment using Imatinib, an abl-kinase inhibitor, has been approved for adult patients with unresectable, recurrent or metastatic DFSP who are not eligible for surgery. The Imatinib results on patients with locally advanced or metastatic disease show variable response, but there are cases with successful results. In particular two cases; a man with DFSP on the thigh and metastasis of the spine and a woman with metastasis in the lungs and axilla show the successful use of imatinib. They were treated with Imatinib for 4 and 3 months, respectively. A tumor size reduction of $75 \%$ was seen in the first case, permitting this patient to surgical resection. In the second case, a CT-scan 3 months after treatment start showed nearly resolution of the lung metastasis. Adjuvant radiotherapy is not commonly preferred, because of reported cases with growth of aggressive fibrosarcomas in areas with DFSP that were treated with radiation therapy. ${ }^{1}$

\section{References}

1. Gloster HM Jr. Dermatofibrsarcoma protuberance. J Am Acad Dermatol 1995;35:355-76.

2. Bague S, Folpe AL. Dermatofibrosarcoma protuberance presenting as a subcutaneous mass: a clinicopathological study of 15 cases with exclusive or near exclusive subcutaneous involvement. Am J Dermatopathol 2008; 30:327-32.

3. Barnes L, Coleman JA, Johnson JT. Dermatofibrosarcoma protuberance of the head and neck. Arch Otolaryngol 1984;110: 398-404.

4. Rockley PF, Robinson JK, Magid M, et al. Dermatofibrosarcoma of the scalp: a series of cases. J Am Acad Dermatol 1989;21:278-83. 